This document is confidential and is proprietary to the American Chemical Society and its authors. Do not copy or disclose without written permission. If you have received this item in error, notify the sender and delete all copies.

\title{
Defect-free self-catalyzed GaAs/GaAsP nanowire quantum dots grown on silicon substrate
}

\begin{tabular}{|c|c|}
\hline Journal: & Nano Letters \\
\hline Manuscript ID & $\mathrm{nl}-2015-04142 \mathrm{n} . \mathrm{R} 1$ \\
\hline Manuscript Type: & Communication \\
\hline Date Submitted by the Author: & 23-Nov-2015 \\
\hline Complete List of Authors: & $\begin{array}{l}\text { Wu, Jiang; University College London, Electronic and Electrical Engineering } \\
\text { Ramsay, Andrew; Hitachi Cambridge Laboratory, Hitachi Europe Ltd. } \\
\text { Sanchez, Ana M.; University of Warwick, Department of Physics } \\
\text { Zhang, Yunyan; University College London, ee } \\
\text { Kim, Dongyoung; University College London, Electronic and Electrical } \\
\text { Engineering } \\
\text { Brossard, F.S.F; Hitachi Cambridge Laboratory, Hitachi Europe Ltd.; Hitachi } \\
\text { Cambridge Laboratory, Hitachi Europe Ltd. } \\
\text { Hu, Xian; University of Arkansas, Physics } \\
\text { Benamara, Mourad; University of Arkansas, Physics } \\
\text { Ware, Morgan; UNIV OF ARKANSAS, Physics } \\
\text { Mazur, Yuriy; University of Arkansas, Department of Physics } \\
\text { Salamo, Gregory; University of Arkansas, } \\
\text { Aagesen, Martin; Gasp Solar ApS } \\
\text { Wang, ZMM; University of Electronic Science and Technology of China } \\
\text { Liu, Huiyun; University College London, Department of Electronic and } \\
\text { Electrical Engineering }\end{array}$ \\
\hline
\end{tabular}




\section{Defect-free self-catalyzed GaAs/GaAsP nanowire}

\section{quantum dots grown on silicon substrate}

Jiang $W^{\dagger, \#, *}$, Andrew Ramsay ${ }^{\ddagger, \#}$, Ana Sanchez ${ }^{\S, \#}$, Yunyan Zhang ${ }^{\dagger, \#}$, Dongyoung Kim ${ }^{\dagger}$, Frederic

Brossard ${ }^{\star}$, Xian Hu\|, Mourad Benamara", Morgan E. Ware", Yuriy I. Mazur\|, Gregory J. Salamo",

Martin Aagesen ${ }^{\perp}$ Zhiming Wang ${ }^{\nabla}$, and Huiyun Liu ${ }^{\dagger, *}$

${ }^{\dagger}$ Department of Electronic and Electrical Engineering, University College London, Torrington Place, London WC1E 7JE, United Kingdom

${ }^{\ddagger}$ Hitachi Cambridge Laboratory, Hitachi Europe Ltd., Cambridge CB3 0HE, United Kingdom

${ }^{\S}$ Department of Physics, University of Warwick, Coventry CV4 7AL, United Kingdom

"Institute for Nanoscience and Engineering, University of Arkansas, Fayetteville, AR 72701, USA

${ }^{\perp}$ Gasp Solar ApS, Gregersensvej 7, Taastrup DK-2630, Denmark

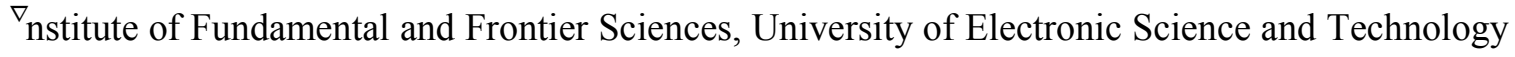
of China, Chengdu 610054, People's Republic of China
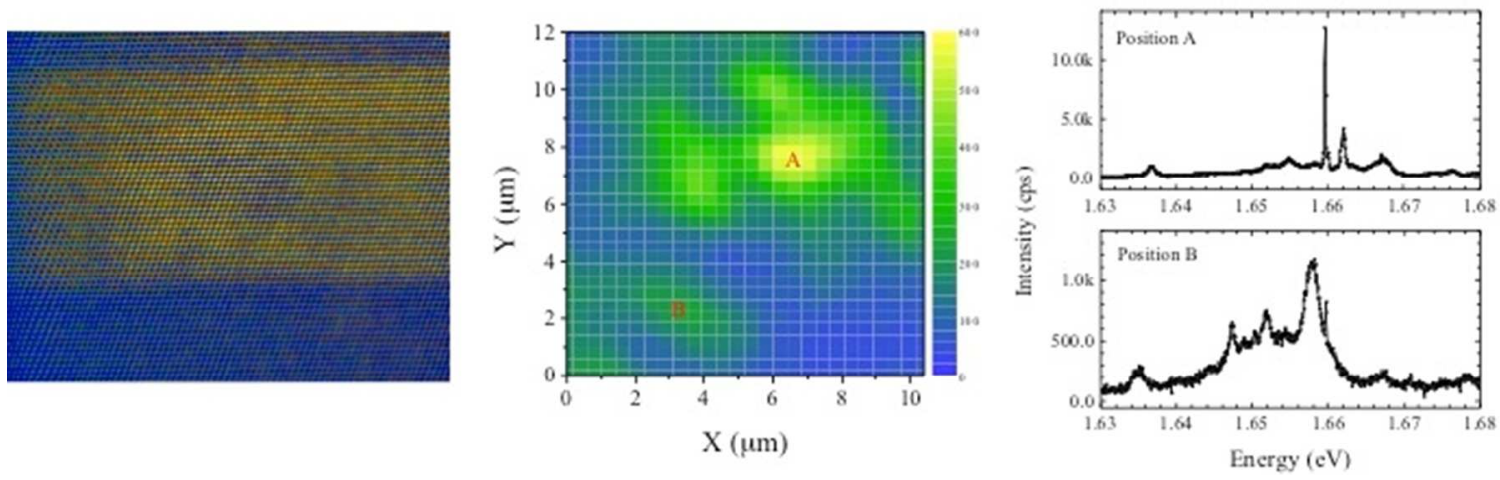

GRAPHIC ABSTRACT 


\begin{abstract}
III-V nanowire quantum dots (NWQDs) monolithically grown on silicon substrates, combining the advantages of both one- and zero-dimensional materials, represent one of the most promising technologies for integrating advanced III-V photonic technologies on a silicon microelectronics platform. However, there are great challenges in the fabrication of high-quality III-V NWQDs by a bottom-up approach, i.e., growth by the vapor-liquid-solid method, because of the potential contamination caused by external metal catalysts and the various types of interfacial defects introduced by self-catalyzed growth. Here, we report the defect-free self-catalyzed III-V NWQDs - GaAs quantum dots in GaAsP nanowires - on a silicon substrate with pure zinc blende structure for the first time. Well-resolved excitonic emission is observed with a narrow linewidth. These results pave the way toward on-chip III-V quantum information and photonic devices on silicon platform.

KEYWORDS: nanowires, quantum dots, self-catalyzed, vapor-liquid-solid, molecular beam epitaxy
\end{abstract}


Semiconductor nanowires by the vapor-liquid-solid (VLS) mechanism offer unique approaches to circumvent the problem of lattice mismatch in thin film heterostructures due to the efficient strain relaxation through the sidewalls and a small footprint ${ }^{1}$. The improved tolerance to lattice mismatch makes nanowires an attractive candidate for monolithically fabricating materials with different lattice constants. For instance, nanowires provide a promising alternative to monolithic integration of III-V materials on silicon substrates ${ }^{2-5}$, which has been pursued for the last few decades $^{6,7}$. In addition, heterojunctions made of materials with distinct different structural and thermal properties can potentially be grown both axially and radially in nanowires virtually free of defects. In this way, nanoscale heterostructures integrating silicon and III-V materials have been reported enriching the functionalities of nanowires ${ }^{8-10}$.

Due to a relaxation of lattice mismatch issues in nanowire structures, thin segments of a narrow band gap material produced in a high band gap nanowire will form nanowire quantum dots (NWQDs), which will open exciting opportunities for applications in emerging fields such as silicon photonics and quantum information ${ }^{11-15}$. Such NWQD structures can significantly boost the light-extraction efficiency to nearly unity and enhance the brightness by up to an order of magnitude higher than conventional quantum dots ${ }^{12,16-18}$. In the last ten years, $\operatorname{In}(\mathrm{As}, \mathrm{P}) / \operatorname{InP}{ }^{19-21}$, (In,Ga)As/GaAs ${ }^{22,}{ }^{23}, \mathrm{CdSe} / \mathrm{ZnSe}^{24}$, and $\mathrm{GaAs} / \mathrm{AlGaAs}{ }^{25}$ NWQDs have been reported on III-V substrates. Meanwhile, NWQDs, consisted of InAsP/InP ${ }^{26}, \mathrm{Ga}(\mathrm{As}, \mathrm{P}) / \mathrm{GaP}^{27-30}, \mathrm{CdSe} / \mathrm{ZnSe}^{31}$, $\mathrm{InGaN} /(\mathrm{Al}, \mathrm{Ga}) \mathrm{N}^{15,32,33}, \mathrm{Ge}(\mathrm{Si}) / \mathrm{Si}^{34,35}$, and $\mathrm{GaAs} / \mathrm{AlGaAs}^{36}$, have also been fabricated on $\mathrm{Si}$ substrates. Despite their relatively recent development, NWQDs have led to a number of significant achievements in quantum information and photonics, including high efficiency and room temperature single photon emitters ${ }^{12,24}$, room temperature lasers ${ }^{37}$, and white LEDs ${ }^{15}$. However, there are a number of problems intrinsic to NWQDs fabricated by the VLS method. Firstly, foreign metal droplets, such as gold nanoparticles, are generally used to catalyze the nanowire growth. However, these catalysts tend to leave trace amounts behind and are considered 
contaminants which are unsuitable for many applications ${ }^{38-40}$. Secondly, it is not a straightforward process to obtain abrupt interfaces in nanowire axial heterostructures using the VLS growth mode. The fabrication of NWQDs involves switching nanowire composition back and forth, during which time it is challenging to maintain the stability of catalyst droplets without changing the interfacial free energies of the catalytic droplets ${ }^{41}$. For the growth of III-V NWQDs on silicon, the requirement of self-catalyzed NWQDs introduces further challenges to obtain high material quality, because the catalyst droplets are directly involved in the nanowire growth. Compared with Au-seeded VLS growth, droplets in self-catalyzed VLS growth can undergo distinct changes of size and volume while varying growth conditions. Lastly, VLS nanowires can have a large number of stacking faults, micro twins, and phase polytypism, which are often observed at the nanowire heterojunction ${ }^{42,43}$. These defects are charge traps and detrimental to the optical and electronic properties of nanowires and NWQDs. Despite the worldwide efforts devoted to this field and striking development during the last few years, there is no report on defect-free NWQDs with sharp interfaces and high phase purity in the literature. Recently, abrupt interfaces have been reported in nanowire heterojunctions, and yet the quality is undermined by the use of gold nanoparticles and the presence of defects such as micro twins and phase polytypism ${ }^{34,44,45}$.

In this paper, regardless of the abovementioned challenges, we report pure zinc-blende GaAs/GaAsP NWQDs fabricated monolithically on silicon substrates by the self-catalyzed VLS method. In addition, an abrupt interface is observed in the nanowire heterojunction that consists of both binary and ternary compounds, which enable a large degree of flexibility in NWQD design and growth. Despite the temporal interruptions during the nanowire growth when switching between binary and ternary materials, the NWQDs show a sharp interface as well as a perfect crystal structure free of any type of defects. Moreover, NWQDs are optically active with narrow excitonic emission spectra, even without surface passivation. 
GaAsP nanowires and GaAs/GaAsP NWQDs were grown on epi-ready Si(111) substrates covered by a thin layer of native oxide. The VLS growth conditions for GaAsP nanowires and GaAs/GaAsP NWQDs were identical except for the GaAs QD region, i.e., the short GaAs segment between GaAsP nanowires as illustrated in Fig. 1a. The Ga-assisted VLS growth of GaAsP nanowires was adopted from earlier work on self-catalyzed GaAsP nanowires ${ }^{46,47}$. For the growth of GaAs QDs, the supply of phosphor was switched off with no growth interrupt in order to avoid any instability of the seed droplets. To compensate the reduced supply of phosphor after switching off, the As beam equivalent pressure (BEP) was increased during the growth of GaAs QDs. After finishing growth of GaAs QDs, the BEPs of As and P molecular beams were switched back to the initial conditions without any interrupt.
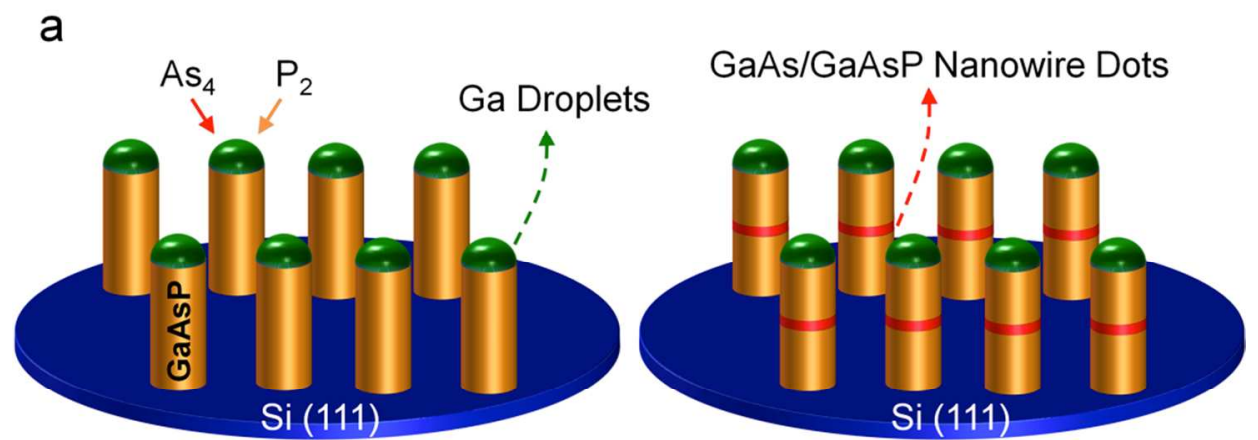

b
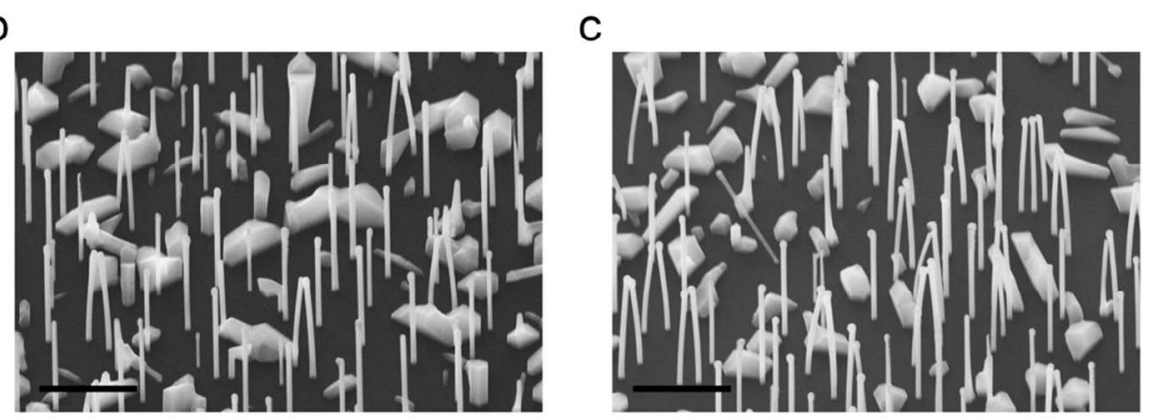

Figure 1. (a) Schematics of the self-catalyzed GaAsP nanowires and GaAs/GaAsP nanowire quantum dots grown by the VLS mechanism. SEM images of (b) GaAsP nanowires, and (c) GaAs/GaAsP nanowire quantum dots. The scale bars in (b) and (c) are $1 \mu \mathrm{m}$. The nanowires are viewed at an angle of $35^{\circ}$ relative to the surface normal. 
Figure $1 \mathrm{~b}$ and $\mathrm{c}$ show the scanning electron microscopy (SEM) images of GaAsP nanowires and GaAs/GaAsP NWQDs taken from a similar position on the wafers. Both samples demonstrate straight nanowires with similar diameters of $\sim 40-50 \mathrm{~nm}$ and length of $\sim 2-4 \mu \mathrm{m}$. The size distribution is caused by the thermal gradient during growth (Fig. S1). Even though each nanowire from the GaAs/GaAsP NWQD sample has a single QD in the middle of the nanowire with two abrupt alterations in growth conditions, no kinking is observed in any of the nanowires in the SEM image. This suggests that the interfacial energetics have been well maintained during the growth of NWQDs. It should be noted that a few NWs were seen to bend and bunch together during observation in the SEM measurements, which is caused by the electrostatic interaction of NWs with accumulated charges induced by the electron beam irradiation.

To evaluate the material composition and interface of single GaAs/GaAsP NWQD, energy-dispersive X-ray (EDX) analysis is used. The data is presented in Fig. 2. It is clearly shown from the low magnification TEM in Fig. 2a that no kinking or distortion of the nanowire is present in the QD region, indicating that inserting a GaAs segment has no significant impact on the stability of the Ga droplets. This is in agreement with the observation of straight NWs from the SEM results in Figure 1. Figure $2 b$ shows the elemental mapping from EDX of a single GaAsP nanowire with a GaAs QD, which distinguishes the two different regions: GaAsP nanowire and GaAs QD. The EDX mapping contrast shows a P-deficient region about $10 \mathrm{~nm}$ in height, which corresponds to the GaAs QD formed in the GaAsP nanowire. The formation of GaAs/GaAsP NWQDs is confirmed by the EDX line scan in Fig. 2c. The atomic percentage profile demonstrates a QD region with higher content of As of 50\% and negligible $\mathrm{P}$ in contrast with the rest of the GaAsP nanowire. The presence of a minute amount of $\mathrm{P}$ in the $\mathrm{QD}$ is due to the small $\mathrm{P}$ background pressure in the MBE growth chamber.

Figure 3a shows a high-resolution angular dark field (ADF) TEM image of the GaAs/GaAsP NWQD. Both GaAs QD and the surrounding GaAsP wire are pure zinc-blende 
structure, without any stacking fault, twinning or polytype observed. Figure $3 \mathrm{~b}$ shows the fast Fourier transform of the high resolution TEM image of Fig. 3a, which confirms again the pure zinc-blende crystal structure and the absence of defects. Two issues determine the quality of the ternary NW heterojunction growth. Firstly, direct removal of the $\mathrm{P}$ beam flux will result in changes to transient supersaturation conditions and interface energetics of droplets, which are responsible for the formation of stacking faults, twin planes, and kinking at the interfaces ${ }^{21,48}$. Secondly, a direct substitution of the reduced P supply with additional As flux of similar BEP can also change the growth landscapes at the interfaces because of the differences in liquid supersaturation free energies, diffusion lengths, incorporation rates, etc. between the two species, $\mathrm{P}$ and $\mathrm{As}^{46,47}$. Particularly, for the self-catalyzed growth mode, the interfacial energetics and droplet supersaturation are vulnerable to minor changes in growth conditions. Any deformation of the droplets can shift the triple phase line, which plays a key role in the phase purity of crystal nucleation $^{49}$. By taking into consideration the combined effects, the effective V/III ratio has been optimized by increasing the As BEP by an amount equal to a few times more than the amount of $\mathrm{P}$ BEP removed during the NWQD growth. In such a way, the interface energetics and droplet supersaturation have been maintained throughout the NWQD growth and we have achieved defect-free GaAsP nanowires with axially embedded QDs similar to QD-free GaAsP nanowires. Only a few defects are scattered at the bottom and tip of the nanowires due to temporal deviation from the optimized growth conditions (Fig. S2). Otherwise, the nanowire is free of any defects and uniform across the entire length of the nanowire, indicating well-balanced $\mathrm{V} / \mathrm{III}$ and $\mathrm{P} / \mathrm{As}$ ratios during the growth (Fig. S2 and Fig. S3).

The two interfaces of the NWQDs at the GaAs and GaAsP boundaries were investigated by high magnification ADF TEM. The images are shown in Fig. 3c and Fig. 3d (false color). The area containing the QD has a higher intensity than the surrounding NW. This is attributed to a higher concentration of heavier elements (As vs. P) in the GaAs QD than in the GaAsP 


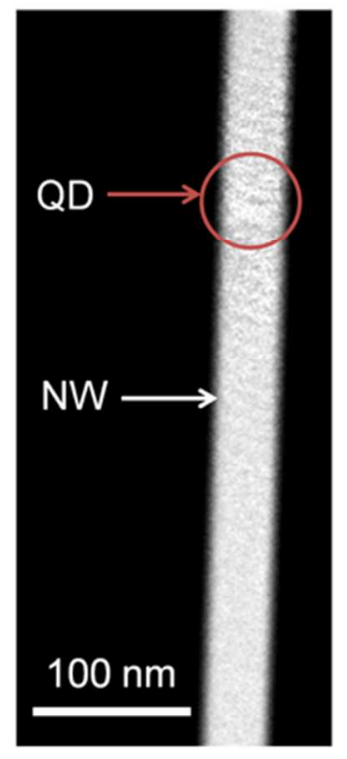

a
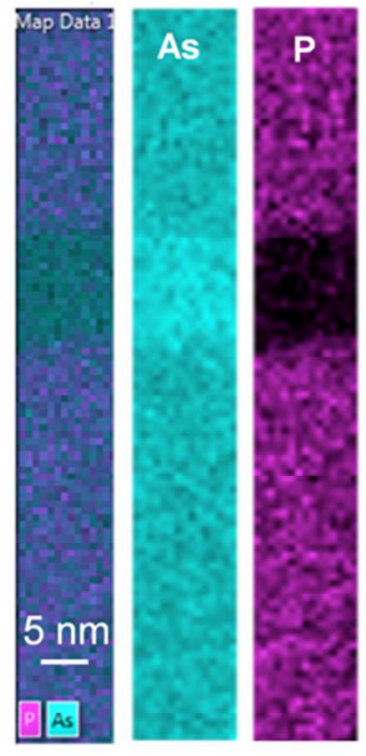

b
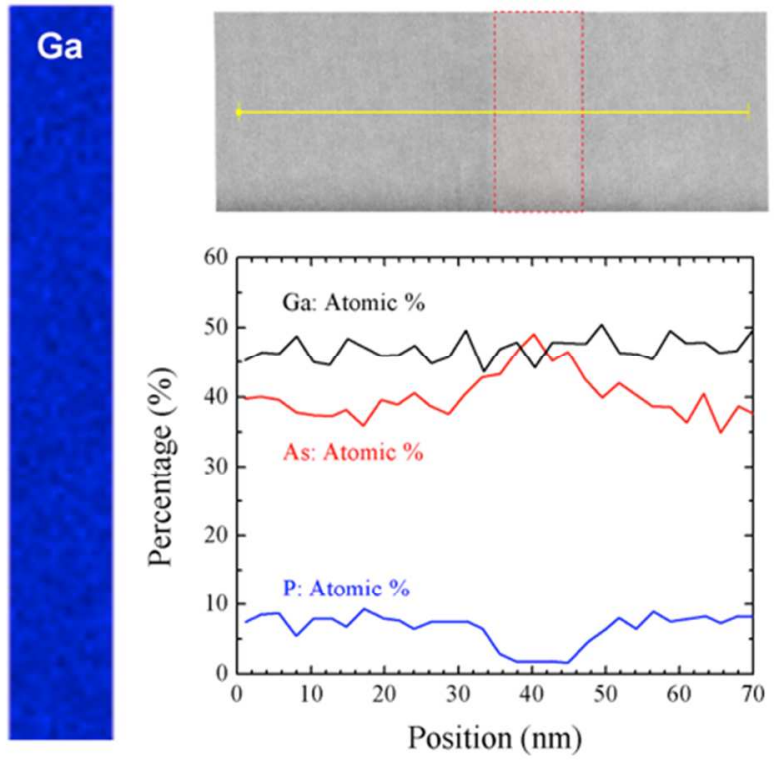

C

Figure 2. (a) Low magnification TEM image of a GaAs QD imbedded in a GaAsP nanowire. (b) EDX mapping of the QD region of the nanowire, which shows a higher content of As and negligible content of P compared with the rest of the nanowire. (c) Element atomic percentage profile taken along the QD area. The yellow line on the low magnification TEM image indicates the position that the EDX measurement is taken.

surroundings. As shown in Fig. 3c and d, the transition from GaAsP to GaAs is completed within a few monolayers and vice versa, in agreement with the EDX measurement of Fig.3a. In Auassisted $\mathrm{GaAs} / \mathrm{GaAsP}$ and $\mathrm{InAsP} / \mathrm{InP}$ interfaces, As carryover can lead to a gradual change in As content and thus non-sharp interfaces ${ }^{14,29}$. In Ref. $\left[{ }^{44}\right]$, flux interruptions were applied to improve the interface sharpness of GaAs segments in $\mathrm{GaP}$ nanowires, but the stability of the catalytic droplets can be impaired and hence twin planes, kinked interfaces, stacking faults, and phase polytypes have been observed. As shown in Fig. 3e and f, both the top and bottom interfaces of the GaAs/GaAsP NWQDs show an abrupt change in contrast in high resolution ADF TEM images, indicating sharp transitions between GaAs and GaAsP sections. The high vapor pressure and lower solubility of $\mathrm{P}$ result in a fast depletion time of $\mathrm{P}$, resulting in the sharp bottom interface during the AsP to As flux transition. On the other hand, As carryover may be less 


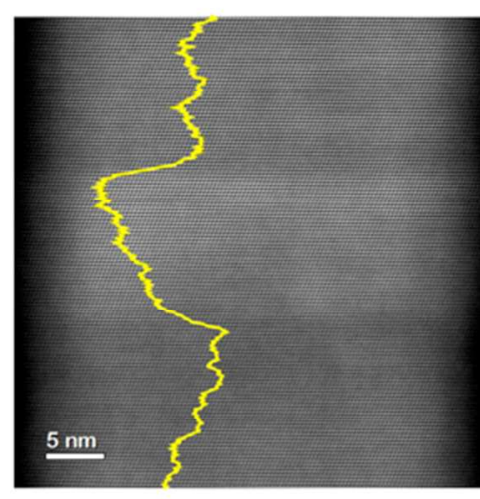

a

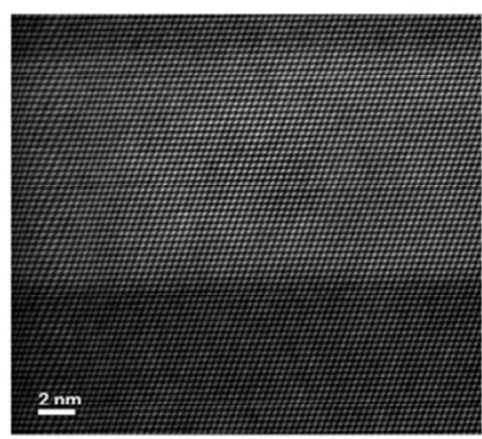

C

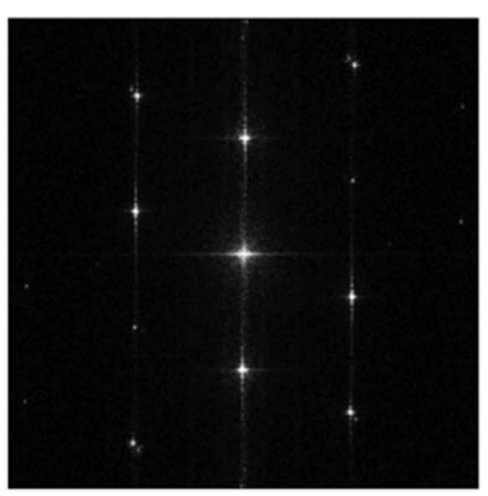

b

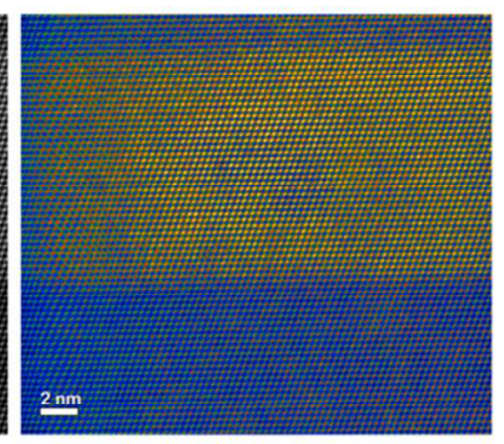

d
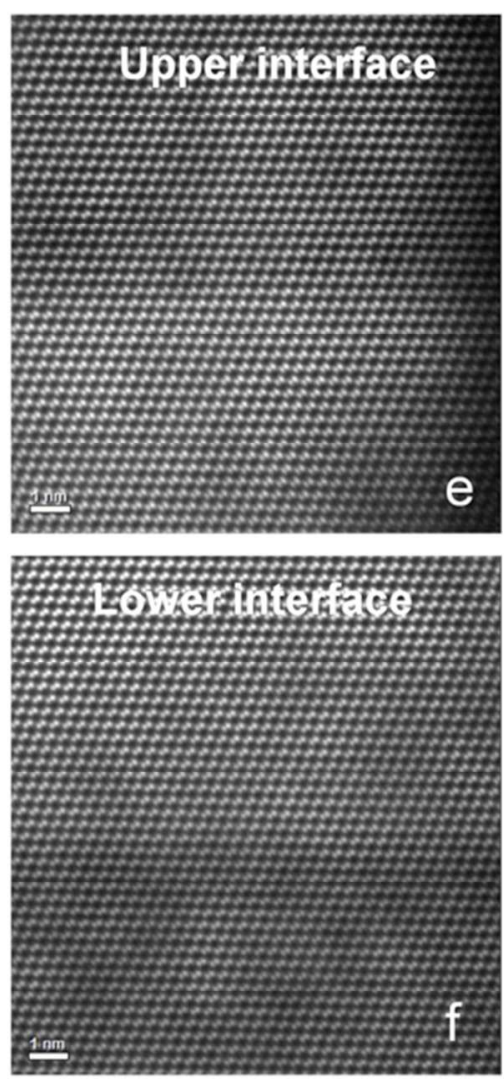

Figure 3. (a) Angular dark field TEM image of the area containing a GaAs QD in the GaAsP nanowire. Both the GaAs QD and the surrounding GaAsP nanowire are zinc-blende structure, without any stacking fault, twinning or polytype. The yellow line is the EDX line scan of the NWQD along the axial axis. (b) Fast Fourier transform of the image in (a), which confirms the pure zinc-blende structure and the absence of defects. (c) High magnification angular dark field image of the GaAs QD, which has a higher intensity ( $\square 10 \mathrm{~nm}$ in height) than the surrounding GaAsP NW. This is an indication of higher concentration of heavier elements (i.e. As vs P) in the QD than in the surroundings. (d) False coloured high magnification angular dark field image of the GaAs QD. (e) and (f) are the high magnification angular dark field image corresponding to the top and bottom interfaces, respectively. No defect is observed and it is a continuous zinc-blende structure. The scale bar in (a) is $5 \mathrm{~nm}$, in (c) and (d) $2 \mathrm{~nm}$, and in (e) and (f) $1 \mathrm{~nm}$. 
Figures $4 \mathrm{a}$ and $\mathrm{b}$ present the normalized photoluminescence (PL) spectra of an ensemble of GaAsP nanowires and GaAs/GaAsP NWQDs measured at $77 \mathrm{~K}$. The GaAsP nanowires show a stable emission peak at $\sim 1.75 \mathrm{eV}(\sim 710 \mathrm{~nm})$ as the excitation power increases. The laser is defocused in order to collect a representative ensemble of nanowires. The excitation power is varied roughly from $600 \mathrm{nW}$ to $6 \mathrm{~mW}$ (power density from 40 to $4 \times 10^{5} \mathrm{~mW} / \mathrm{cm}^{2}$ ). At $77 \mathrm{~K}$, the emission energy corresponds to $\mathrm{GaAs}_{0.8} \mathrm{P}_{0.2}$ bandgap (Fig. S4). The broad emission peak (FWHM 53 meV) indicates a large distribution (size, composition) of the GaAsP nanowires due to random nucleation of the self-catalyzed ternary nanowires. Various types of surface states and defects may also be attributed to the broad emission because no passivation layer is applied on the nanowires $^{50}$.

For the NWQD sample, (Fig. 4b), at low excitation power a narrower ( $\sim 34 \mathrm{meV}$ vs $\sim 53$ meV) linewidth peak is observed at $1.57 \mathrm{eV}$. This is between the bandgaps of $\mathrm{GaAs}_{0.98} \mathrm{P}_{0.02}$ and $\mathrm{GaAs}_{0.8} \mathrm{P}_{0.2}$, and is consistent with emission from the ground-state of a GaAs quantum dot. In addition, the narrower linewidth is consistent with quantum dots, whose energies are more tolerant to composition variation. A distinct blue-shift is observed with increasing excitation power. A similar PL blue shift has been observed in zinc-blend/wurtzite phase superlattices ${ }^{51}$. However, mixed crystal phase has not been observed in our NWQDs. Therefore, we attribute the blue-shift to state-filling of the quantum dot excited states, that are unresolved due to inhomogeneous broadening of NWQDs with different sizes and compositions. At the highest excitation power, the PL spectrum again shows a peak at $\sim 1.75 \mathrm{eV}$ due to the recombination in the GaAsP region of the GaAsP/GaAs NWQDs. It should be noted that a broad shoulder appears around 1.6-1.68 eV in the PL spectrum of GaAsP nanowire sample. This is likely due to the surface effects of the unpassivated nanowires. In addition, as shown in Fig. S5 and Table S1, the clusters formed from the parasitic two-dimensional growth show large composition variation and 
a large number of defects, which could result in the observation of the below-bandgap shoulder in the PL spectra.
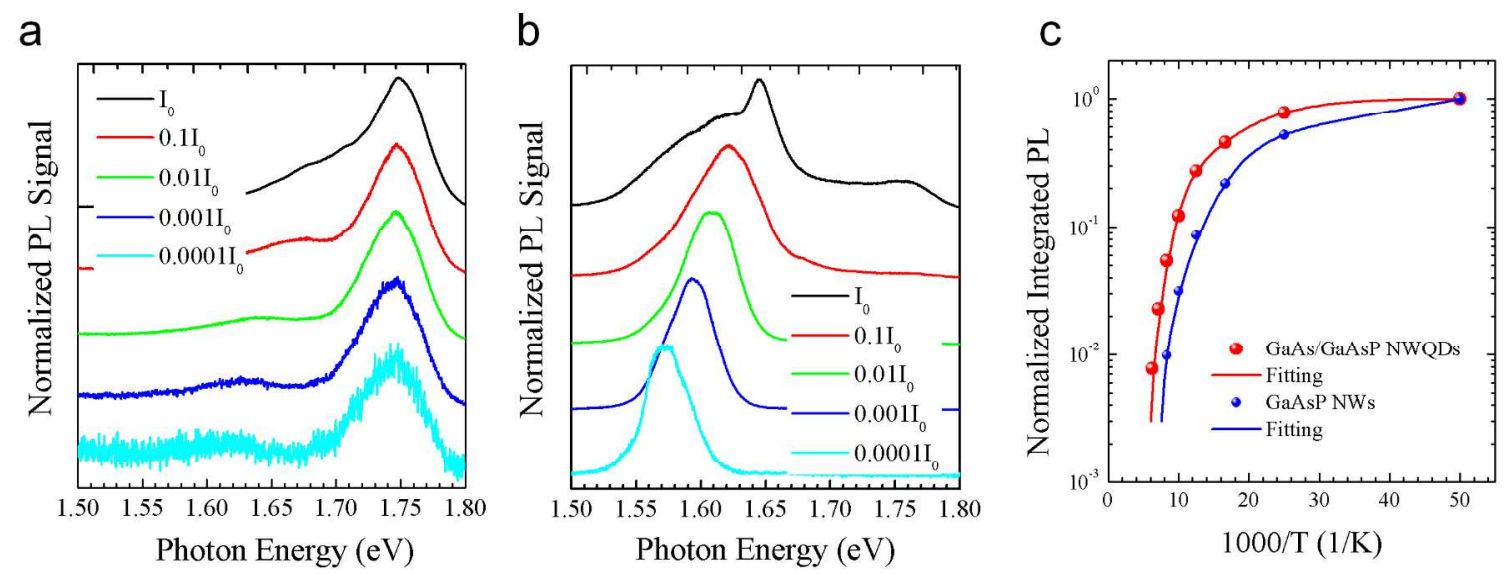

Figure 4. Normalized power-dependent micro-PL spectra measured at $77 \mathrm{~K}$ : (a) GaAsP nanowires and (b) GaAs/GaAsP NWQDs. The excitation was defocused and thus, emission from ensembles of nanowires was collected. The excitation power $\mathrm{I}_{0}$ is $\sim 6 \mathrm{~mW}$. (c) Temperature-dependent integrated PL intensities for GaAsP nanowires (blue circles) and GaAs/GaAsP NWQDs (red circles). The integrated PL intensities are normalized by the values measured at $\mathrm{T} \approx 20 \mathrm{~K}$ for each sample. The temperature-dependent integrated PL intensities are fitted by using the dual-Arrhenius equation.

To gain further insight into the electronic structure and surface effects of the nanowires, temperature dependent PL are taken, see Fig. S6 and Table S2. Figure 4c plots the integrated PL intensity for the GaAsP nanowires (blue circles) and GaAs/GaAsP NWQDs (red circles). The PL quenching is characterized by two activation energies, $\mathrm{E}_{\mathrm{a} 1}$ and $\mathrm{E}_{\mathrm{a} 2}$. For GaAsP nanowires, $\mathrm{E}_{\mathrm{a} 1}=$ $1.9 \pm 1.3 \mathrm{meV}$, is attributed to thermal-excitation of photo-carriers from weakly localised states caused by composition fluctuation. The $\mathrm{E}_{\mathrm{a} 1}$ value of the GaAs/GaAsP NWQDs, $14.3 \pm 0.4 \mathrm{meV}$, is similar to the value obtained from unpassivated GaAs nanowires and is associated with the GaAs surface states ${ }^{52}$. By comparison, for the GaAsP NW sample, $\mathrm{E}_{\mathrm{a} 2}=27.3 \pm 8.1 \mathrm{meV}$, is also attributed to surface non-radiative recombination, but is higher due to the reduced surface recombination velocity of GaAsP in comparison with GaAs. The high temperature PL quenching 
of GaAs/GaAsP NWQDs yields an activation energy of $\mathrm{E}_{\mathrm{a} 2}=73.4 \pm 5.3 \mathrm{meV}$. This can be attributed to thermal excitation of photo-carriers from the GaAs QDs into the GaAsP barriers. This suggests that the GaAsP barriers provide a sufficient confinement potential but the surface states of the GaAs limits the optical properties of the nanowires.

The optical properties of individual GaAsP NWs and GaAs/GaAsP NWQDs were further characterized by micro-PL ( $\mu$-PL) spectroscopy at $5 \mathrm{~K}$. The laser spot diameter is about $1.8 \mu \mathrm{m}$ and excitation power is varied from about $2.5 \mathrm{nW}$ up to $1800 \mathrm{nW}$ (power density from 40 to $1.8 \times 10^{4} \mathrm{~mW} / \mathrm{cm}^{2}$ ). The PL spectra of a representative single GaAsP NW are shown in Fig. 5a. No band edge emission is observed from GaAsP nanowires (about $1.76 \mathrm{eV}$ at $5 \mathrm{~K}$ for $20 \% \mathrm{P}$ ). However, well-resolved emission peaks are present below the GaAsP bandgap. These rather broad emission peaks can be found in an energy range from $1.62 \mathrm{eV}$ to $1.66 \mathrm{eV}$ for different nanowires. In addition to the different emission positions, there is also large variation between wires in the spectral shape and line width. As shown in Fig. S7, the full width at half maximum (FWHM) of a single nanowire can be as broad as $\sim 20 \mathrm{meV}$ but some wires have a relatively sharp line with FWHM of $\sim 1-2 \mathrm{meV}$. The broad emission peaks can be observed in a wide range of excitation as well as at higher temperatures (Fig. 5b). These broad peaks match well the shoulder observed in the PL spectra from the ensemble of nanowires at $77 \mathrm{~K}$, and thus the broad emission peaks are attributed to the surface states. In contrast, the sharp peaks disappear at high excitation power leaving only the broad peaks. This suggests that the narrow emission peaks originate from localized states. At high excitation power, the localized states are filled and emission only takes place through the surface states. This is also in agreement with the temperature-dependent measurement, showing that the narrow PL peaks quench rapidly with increasing temperature (Fig. 5b). Although the nanowires show high crystal quality and uniform composition distribution in nearly the entire wire, the bottom and tip of the nanowires both have a high density of stacking faults and larger composition variation, which may be responsible for the 
localized states (Fig. S2). In addition, the parasitic clusters formed near the nanowires cannot be ruled out for the narrow emission peaks observed in the single nanowire measurements.

Fig. 5c shows the emission spectrum measured from a single GaAs/GaAsP NWQD. The NWQD shows sharp single exciton peak (and possible biexciton as well) at $\sim 1.66 \mathrm{eV}$. Although no shell or passivation layer have been grown over the NWQDs, the emission line width is measured as narrow as $130 \mu \mathrm{eV}$ at $5 \mathrm{~K}$, approaching the equipment resolution limit. This value is comparable to best reported values of the Au-seeded NWQDs with protection shells ${ }^{20,30}$. Most of the NWQDs show narrow emission peaks with FWHM about $0.5 \mathrm{meV}$, where the nearby surface states may be the major reason for the inhomogeneous broadening (Fig. S8). The charged surface states of the unpassivated nanowires can cause spectral diffusion and enhance the emission line width $^{53}$. For example, the surface oxide can have a significant impact on the QD emission (Fig. S9). The observation of some of the slightly broader peaks can also be due to unresolved additional peaks from neighboring nanowires (Fig. S8 and Fig. S10).

For all of the GaAs/GaAsP NWQDs measured, at low excitation powers, the emission is dominated by bright narrow lines with peak emission counts over 10kcps, which is bright compared with S-K QDs measured in the same setup. This indicates good radiative efficiency of the NWQDs, and that the GaAs/GaAsP QD is consistently the lowest energy state of the NWQD system. By contrast, at low powers there is a large variation in the spectra of GaAsP NWs. Most have broad spectra. On occasion, the GaAsP NWs exhibit relatively narrow $\sim 2 \mathrm{meV}$ lines (Fig. S7), but where the emission is dominated by additional broad spectral features, and quenched at high powers. This implies low radiative efficiency, consistent with localized surface states. In a small number of GaAsP NWs, a single line of similar linewidth to the GaAs/GaAsP NWQD is observed. However, in sharp contrast to broad and low emission efficiency of the GaAsP nanowires, in GaAs/GaAsP NWQD, the emission is mostly into the narrow lines. At high excitation powers, the NWQD shows a rich spectrum with additional sharp emission features 
which are not presented in GaAsP NWs. In the NWQD, the group of narrow lines is consistent with charged exciton or multiple excitons implying a strong confinement of the Coulomb-shifted carriers, and QD like character, whereas the GaAsP nanowires exhibit only one narrow line consistent with shallow localized confinement. In the NWQD, the additional sharp lines on both sides of the neutral exciton line are attributed to different charged exciton species. Fig. 5d shows the temperature-dependent PL spectra of a single NWQD. The sharp emission lines show rapid thermal quenching because of the shallow hole confinement. From the PL measurements of GaAsP nanowires without QDs, the surface states show energy transitions in similar energy range, and when such surface states are present in the GaAsP barrier near the NWQDs, they may also cause the rapid PL quenching of the NWQDs. Nevertheless, the narrow lines of GaAs/GaAsP NWQD are more robust against power and temperature compared with GaAsP NW, suggesting deeper confinement and better radiative efficiency.

We further assess the origin of the emission peaks of the GaAs/GaAsP NWQDs by $\mu$-PL intensity mapping as presented in Fig. 5e. The emission signals are collected over the entire spectral range covering all of the observed PL peaks. Fig. 5e clearly shows that there are emission signals collected away from the NWQD. Fig. $5 f$ shows the PL spectra measured in positions A and $\mathrm{B}$ as indicated in Fig. 5e. The strong and narrow emission from position A indicates the formation of high quality NWQDs. Away from the NWQD, a complex spectrum of about one order of magnitude weaker in intensity is measured from position B, which supports the speculation of the background emission with low intensity and broad features around 1.62-1.66 $\mathrm{eV}$ from the parasitic clusters. 

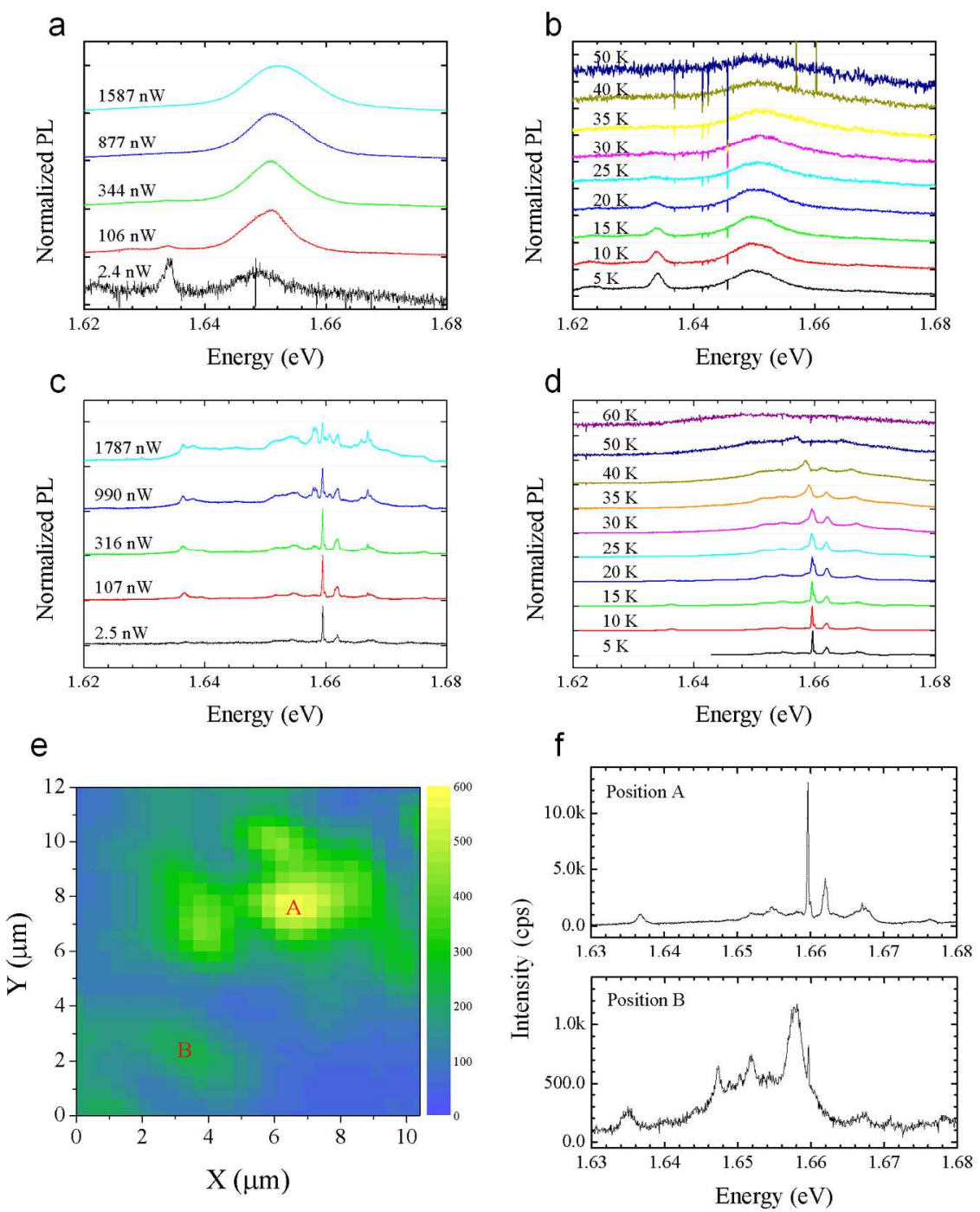

Figure 5. (a) Normalized $\mu$-PL spectra of a single GaAsP nanowire under different excitation powers at $5 \mathrm{~K}$. (b) Temperature-dependent PL spectra of the single GaAsP nanowire. (c) Normalized $\mu$-PL spectra of a single GaAs/GaAsP NWQD under different excitation powers at 5 K. (d) Temperature-dependent PL spectra of the single GaAs/GaAsP NWQD. (e) $12 \times 10 \mu \mathrm{m}^{2}$ integrated $\mu$-PL intensity map of NWQDs at $5 \mathrm{~K}$. The $\mu$-PL signal is integrated over the energy range between 1.62 and $1.67 \mathrm{eV}$. (f) Individual $\mu$-PL spectra measured at the position A and B from the $\mu$-PL intensity map, respectively.

In conclusion, we demonstrate that defect-free NWQDs can be obtained by using the selfcatalyzed VLS growth method. The self-catalyzed NWQDs minimize the incorporation of contaminants, such as foreign metals, and show promises for quantum devices requiring high 
material quality, such as single photon emitters. Without any surface protection layer, an exciton emission line width as narrow as $130 \mu \mathrm{eV}$ has been achieved in this paper. Therefore, these NWQDs have great potential in the field of quantum information and nanophotonics. For example, these NWQDs are expected to achieve low-threshold nanolasers on Si substrates. This method of achieving defect-free and sharp axial heterostructures in nanowires also opens new possibilities for engineering novel functional nanowire architectures, such as QD molecules.

\section{ASSOCIATED CONTENT}

\section{Supporting Information}

Further details of experimental methods and measurements. This material is available free of charge via the Internet at http://pubs.acs.org

\section{AUTHOR INFORMATION}

Corresponding Author

*E-mail: jiang.wu@ucl.ac.uk (J.W.), huiyun.liu@ucl.ac.uk (H.L.)

\section{Notes}

\# These authors contributed equally to this work.

\section{ACKNOWLEDGMENT}

The authors acknowledge the support of Leverhulme Trust (UK), UK EPSRC under Grant No. EP/K004077/1, and the National Science Foundation of the U.S. (Grant \# DMR-1309989). H.L. would like to thank The Royal Society for funding his University Research Fellowship.

\section{REFERENCES}

1. Ertekin, E.; Greaney, P. A.; Chrzan, D.; Sands, T. D. J. Appl. Phys. 2005, 11, 114325. 
2. Mårtensson, T.; Svensson, C. P. T.; Wacaser, B. A.; Larsson, M. W.; Seifert, W.; Deppert, K.; Gustafsson, A.; Wallenberg, L. R.; Samuelson, L. Nano Lett. 2004, 10, 1987-1990.

3. Chuang, L. C.; Sedgwick, F. G.; Chen, R.; Ko, W. S.; Moewe, M.; Ng, K. W.; Tran, T. D.; Chang-Hasnain, C. Nano Lett. 2010, 2, 385-390.

4. Chen, R.; Tran, T. D.; Ng, K. W.; Ko, W. S.; Chuang, L. C.; Sedgwick, F. G.; Chang-Hasnain, C. Nature Photon. 2011, 3, 170-175.

5. Wu, J.; Li, Y.; Kubota, J.; Domen, K.; Aagesen, M.; Ward, T.; Sanchez, A.; Beanland, R.; Zhang, Y.; Tang, M. Nano Lett. 2014, 4, 2013-2018.

6. Liu, H.; Wang, T.; Jiang, Q.; Hogg, R.; Tutu, F.; Pozzi, F.; Seeds, A. Nat Photon 2011, 7, 416419.

7. Chen, S.; Tang, M.; Wu, J.; Jiang, Q.; Dorogan, V.; Benamara, M.; Mazur, Y.; Salamo, G.; Seeds, A.; Liu, H. Electron. Lett. 2014, 20, 1467-1468.

8. Hocevar, M.; Immink, G.; Verheijen, M.; Akopian, N.; Zwiller, V.; Kouwenhoven, L.; Bakkers, E. Nature communications 2012, 3, 1266.

9. Conesa-Boj, S.; Hauge, I.; Verheijen, M. A.; Assali, S.; Li, A.; Bakkers, E. P.; Fontcuberta i Morral, A. Nano letters 2015, 15, 2974-2979.

10. Conesa-Boj, S.; Boioli, F.; Russo-Averchi, E.; Dunand, S.; Heiss, M.; Rüffer, D.; Wyrsch, N.; Ballif, C.; Miglio, L.; Morral, A. F. i. Nano letters 2014, 4, 1859-1864.

11. Hu, Y.; Churchill, H. O.; Reilly, D. J.; Xiang, J.; Lieber, C. M.; Marcus, C. M. Nature nanotechnology 2007, 10, 622-625. 
12. Reimer, M. E.; Bulgarini, G.; Akopian, N.; Hocevar, M.; Bavinck, M. B.; Verheijen, M. A.; Bakkers, E. P.; Kouwenhoven, L. P.; Zwiller, V. Nature communications 2012, 3, 737.

13. Björk, M. T.; Thelander, C.; Hansen, A. E.; Jensen, L. E.; Larsson, M. W.; Wallenberg, L. R.; Samuelson, L. Nano Letters 2004, 9, 1621-1625.

14. Minot, E. D.; Kelkensberg, F.; Van Kouwen, M.; Van Dam, J. A.; Kouwenhoven, L. P.; Zwiller, V.; Borgström, M. T.; Wunnicke, O.; Verheijen, M. A.; Bakkers, E. P. Nano letters 2007, 2, 367-371.

15. Nguyen, H. P. T.; Zhang, S.; Cui, K.; Han, X.; Fathololoumi, S.; Couillard, M.; Botton, G.; Mi, Z. Nano letters 2011, 5, 1919-1924.

16. Uccelli, E.; Arbiol, J.; Morante, J. R.; Fontcuberta i Morral, A. ACS nano 2010, 10, 59855993.

17. Claudon, J.; Bleuse, J.; Malik, N. S.; Bazin, M.; Jaffrennou, P.; Gregersen, N.; Sauvan, C.; Lalanne, P.; Gérard, J. Nature Photonics 2010, 3, 174-177.

18. Bleuse, J.; Claudon, J.; Creasey, M.; Malik, N. S.; Gérard, J.; Maksymov, I.; Hugonin, J.; Lalanne, P. Phys. Rev. Lett. 2011, 10, 103601.

19. Fuhrer, A.; Fröberg, L. E.; Pedersen, J. N.; Larsson, M. W.; Wacker, A.; Pistol, M.; Samuelson, L. Nano letters 2007, 2, 243-246.

20. Dalacu, D.; Mnaymneh, K.; Lapointe, J.; Wu, X.; Poole, P. J.; Bulgarini, G.; Zwiller, V.; Reimer, M. E. Nano letters 2012, 11, 5919-5923. 
21. Tchernycheva, M.; Cirlin, G. E.; Patriarche, G.; Travers, L.; Zwiller, V.; Perinetti, U.; Harmand, J. Nano letters 2007, 6, 1500-1504.

22. Tatebayashi, J.; Ota, Y.; Ishida, S.; Nishioka, M.; Iwamoto, S.; Arakawa, Y. Appl. Phys. Lett. 2014, 10, 103104.

23. Panev, N.; Persson, A. I.; Sköld, N.; Samuelson, L. Appl. Phys. Lett. 2003, 11, 2238-2240.

24. Bounouar, S.; Elouneg-Jamroz, M.; Hertog, M. d.; Morchutt, C.; Bellet-Amalric, E.; André, R.; Bougerol, C.; Genuist, Y.; Poizat, J.; Tatarenko, S. Nano letters 2012, 6, 2977-2981.

25. Heinrich, J.; Huggenberger, A.; Heindel, T.; Reitzenstein, S.; Höfling, S.; Worschech, L.; Forchel, A. Appl. Phys. Lett. 2010, 21, 211117.

26. Kuyanov, P.; LaPierre, R. Nanotechnology 2015, 31, 315202.

27. Verheijen, M. A.; Immink, G.; de Smet, T.; Borgström, M. T.; Bakkers, E. P. J. Am. Chem. Soc. 2006, 4, 1353-1359.

28. Gudiksen, M. S.; Lauhon, L. J.; Wang, J.; Smith, D. C.; Lieber, C. M. Nature 2002, 6872, 617-620.

29. Borgström, M. T.; Verheijen, M. A.; Immink, G.; de Smet, T.; Bakkers, E. P. Nanotechnology 2006, 16, 4010.

30. Borgström, M. T.; Zwiller, V.; Müller, E.; Imamoglu, A. Nano letters 2005, 7, 1439-1443.

31. Tribu, A.; Sallen, G.; Aichele, T.; Andre, R.; Poizat, J.; Bougerol, C.; Tatarenko, S.; Kheng, K. Nano letters 2008, 12, 4326-4329. 
32. Deshpande, S.; Frost, T.; Yan, L.; Jahangir, S.; Hazari, A.; Liu, X.; Mirecki-Millunchick, J.; Mi, Z.; Bhattacharya, P. Nano letters 2015, 3, 1647-1653.

33. Deshpande, S.; Das, A.; Bhattacharya, P. Appl. Phys. Lett. 2013, 16, 161114.

34. Wen, C. Y.; Reuter, M. C.; Bruley, J.; Tersoff, J.; Kodambaka, S.; Stach, E. A.; Ross, F. M. Science 2009, 5957, 1247-1250.

35. Wu, Y.; Fan, R.; Yang, P. Nano Letters 2002, 2, 83-86.

36. Heiss, M.; Fontana, Y.; Gustafsson, A.; Wüst, G.; Magen, C.; O’Regan, D.; Luo, J.; Ketterer, B.; Conesa-Boj, S.; Kuhlmann, A. Nature materials 2013, 5, 439-444.

37. Tatebayashi, J.; Kako, S.; Ho, J.; Ota, Y.; Iwamoto, S.; Arakawa, Y. Nature Photonics 2015, 9, 501-505.

38. Bar-Sadan, M.; Barthel, J.; Shtrikman, H.; Houben, L. Nano letters 2012, 5, 2352-2356.

39. Oh, S. H.; Benthem, K. v.; Molina, S. I.; Borisevich, A. Y.; Luo, W.; Werner, P.; Zakharov, N. D.; Kumar, D.; Pantelides, S. T.; Pennycook, S. J. Nano letters 2008, 4, 1016-1019.

40. Allen, J. E.; Hemesath, E. R.; Perea, D. E.; Lensch-Falk, J. L.; Li, Z.; Yin, F.; Gass, M. H.; Wang, P.; Bleloch, A. L.; Palmer, R. E. Nature Nanotechnology 2008, 3, 168-173.

41. Dick, K. A.; Kodambaka, S.; Reuter, M. C.; Deppert, K.; Samuelson, L.; Seifert, W.; Wallenberg, L. R.; Ross, F. M. Nano letters 2007, 6, 1817-1822.

42. Dheeraj, D. L.; Patriarche, G.; Zhou, H.; Hoang, T. B.; Moses, A. F.; Grønsberg, S.; van Helvoort, A. T.; Fimland, B.; Weman, H. Nano letters 2008, 12, 4459-4463. 
43. Patriarche, G.; Glas, F.; Tchernycheva, M.; Sartel, C.; Largeau, L.; Harmand, J.; Cirlin, G. E. Nano letters 2008, 6, 1638-1643.

44. Priante, G.; Patriarche, G.; Oehler, F.; Glas, F.; Harmand, J. Nano Lett. 2015,15, 6036-6041.

45. Lehmann, S.; Wallentin, J.; Jacobsson, D.; Deppert, K.; Dick, K. A. Nano letters 2013, 9, 4099-4105.

46. Holm, J. V.; Jørgensen, H. I.; Krogstrup, P.; Nygård, J.; Liu, H.; Aagesen, M. Nature communications 2013, 4, 1498.

47. Zhang, Y.; Aagesen, M.; Holm, J. V.; Jørgensen, H. I.; Wu, J.; Liu, H. Nano letters 2013, 8, 3897-3902.

48. Johansson, J.; Dick, K. A. CrystEngComm 2011, 24, 7175-7184.

49. Yu, X.; Wang, H.; Lu, J.; Zhao, J.; Misuraca, J.; Xiong, P.; von Molnár, S. Nano Lett. 2012, $5436-5442$.

50. Sun, M.; Joyce, H.; Gao, Q.; Tan, H.; Jagadish, C.; Ning, C. Nano letters 2012, 7, 3378-3384.

51. Bao, J.; Bell, D. C.; Capasso, F.; Wagner, J. B.; Mårtensson, T.; Trägårdh, J.; Samuelson, L. Nano letters 2008, 3, 836-841.

52. Couto Jr, O.; Sercombe, D.; Puebla, J.; Otubo, L.; Luxmoore, I.; Sich, M.; Elliott, T.;

Chekhovich, E.; Wilson, L.; Skolnick, M. Nano letters 2012, 10, 5269-5274.

53. Seufert, J.; Weigand, R.; Bacher, G.; Kümmell, T.; Forchel, A.; Leonardi, K.; Hommel, D. Appl. Phys. Lett. 2000, 14, 1872-1874. 


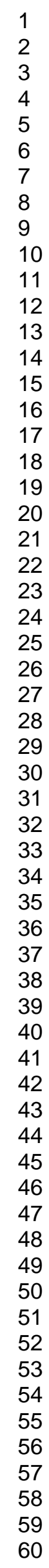



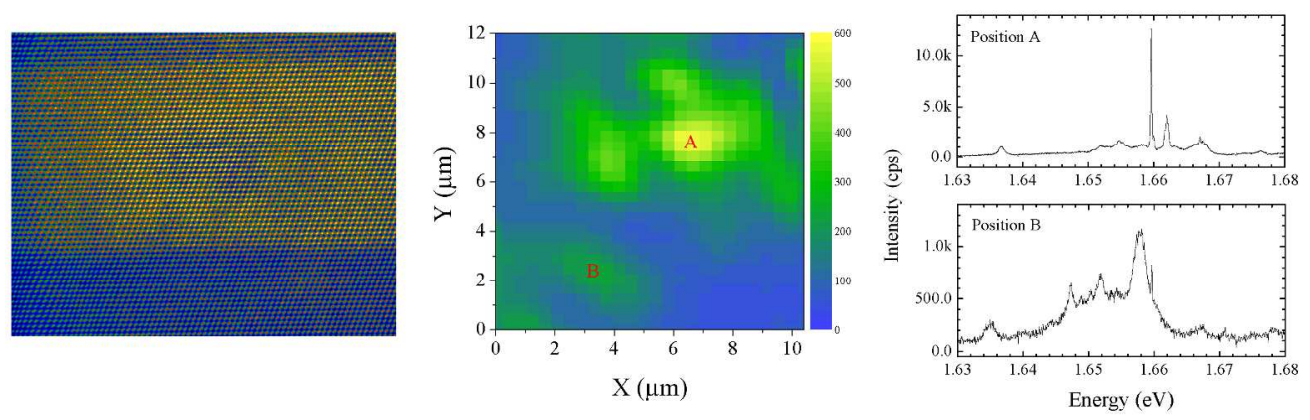

17

Graphic abstract $244 \times 75 \mathrm{~mm}$ (300 x 300 DPI) 

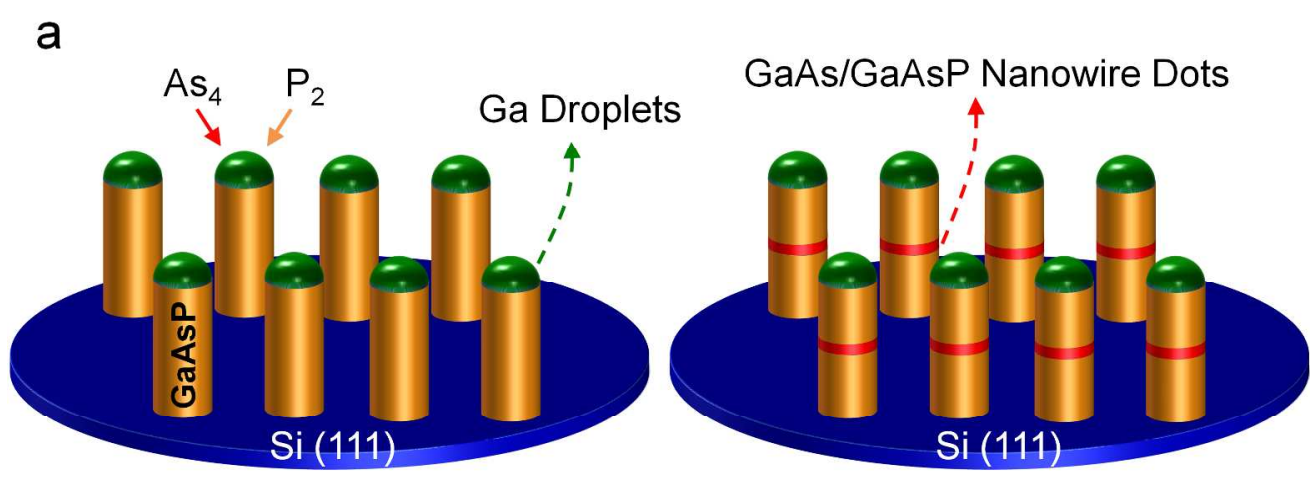

b

C
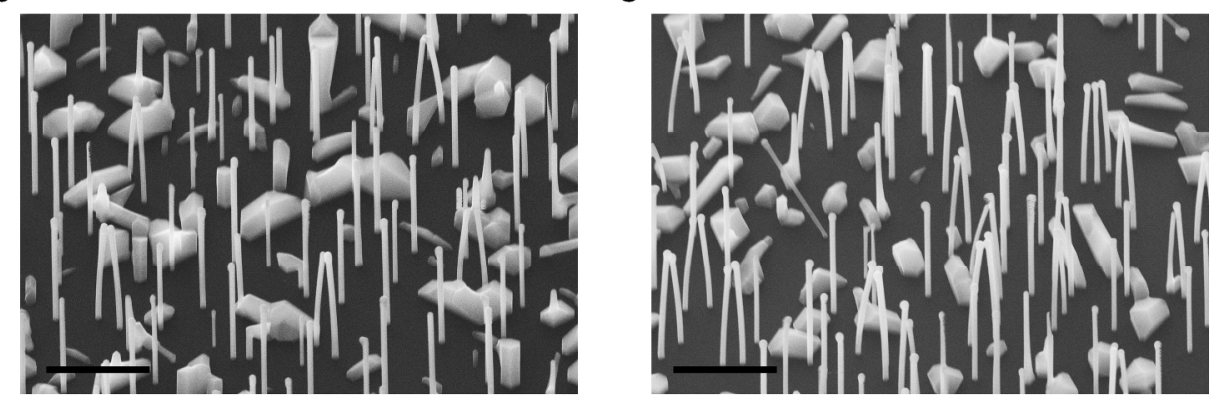

Figure 1

$1387 \times 996 \mathrm{~mm}$ ( 55 x 55 DPI) 


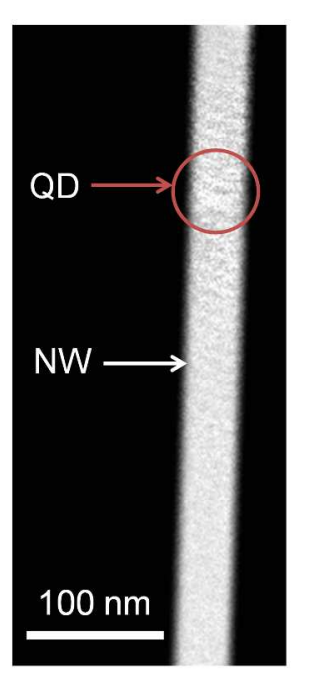

a

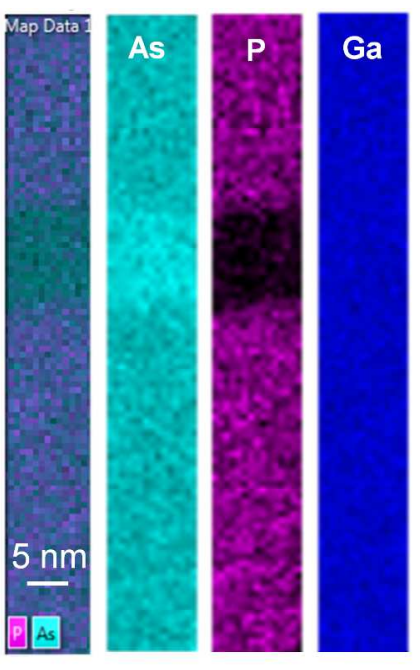

b
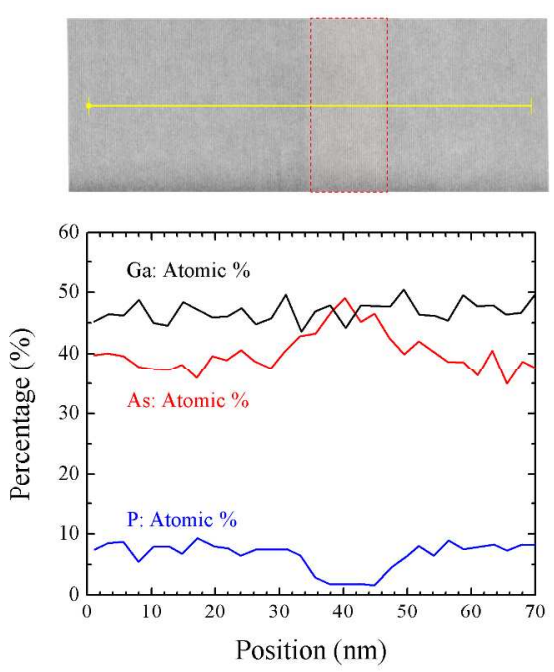

C

Figure 2

$241 \times 133 \mathrm{~mm}(300 \times 300$ DPI $)$ 


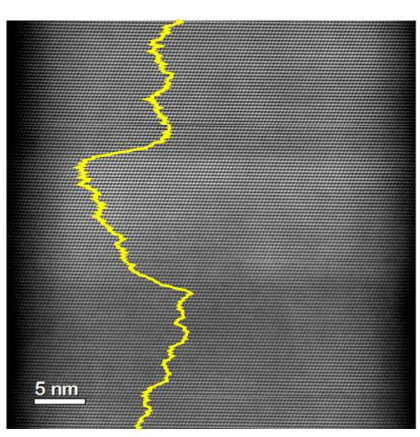

a

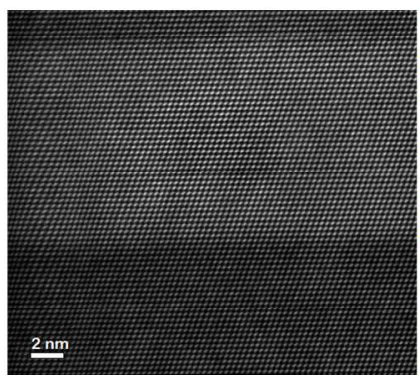

C

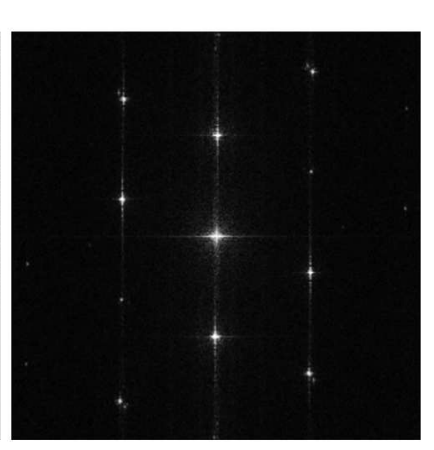

b

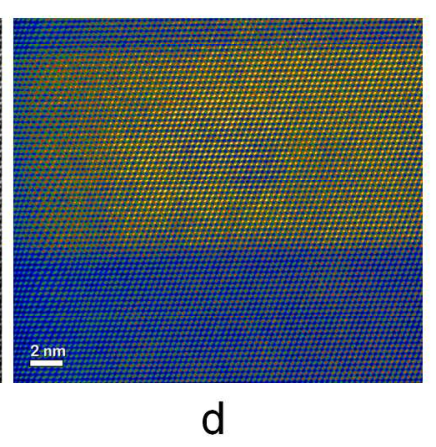

Figure 3
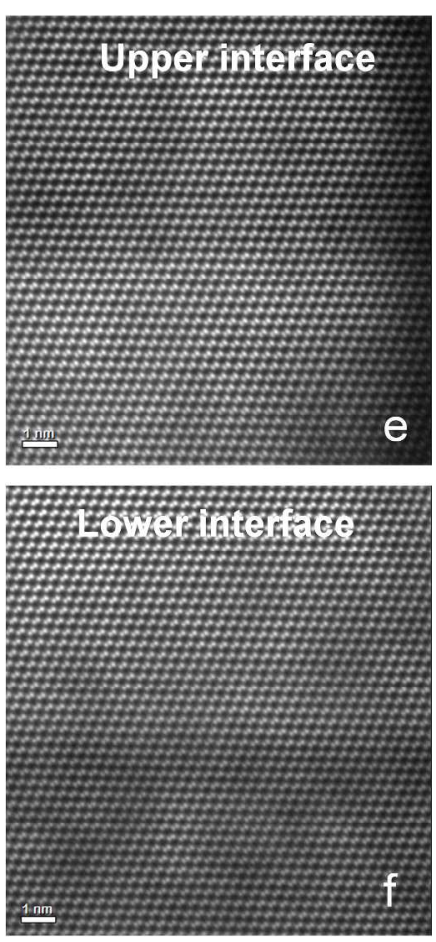

$242 \times 176 \mathrm{~mm}(300 \times 300$ DPI $)$ 
1

2

3

4

5

6

8

9

10

11

12

13

14

15

16

17

18

19

20

21

22

23

24

25

26

27

28

29

30

31

32

33

34

35

36

37

38

39

40

41

42

43

44

45

46

47

48

49

50

51

52

53

54

55

56

57

58

59

60

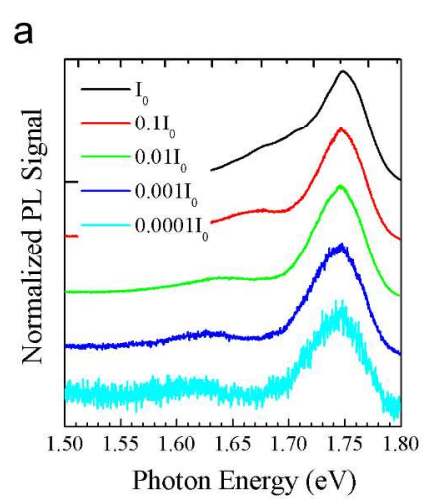

b
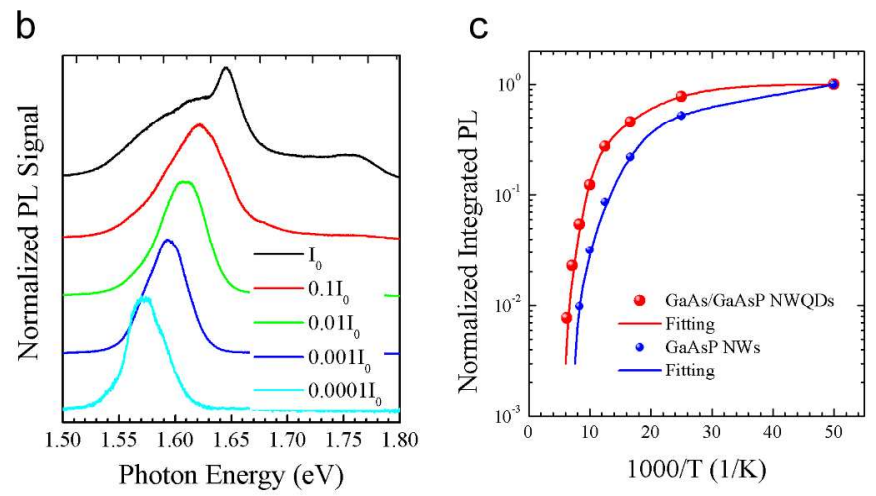

Figure 4

$213 \times 82 \mathrm{~mm}(300 \times 300 \mathrm{DPI})$ 

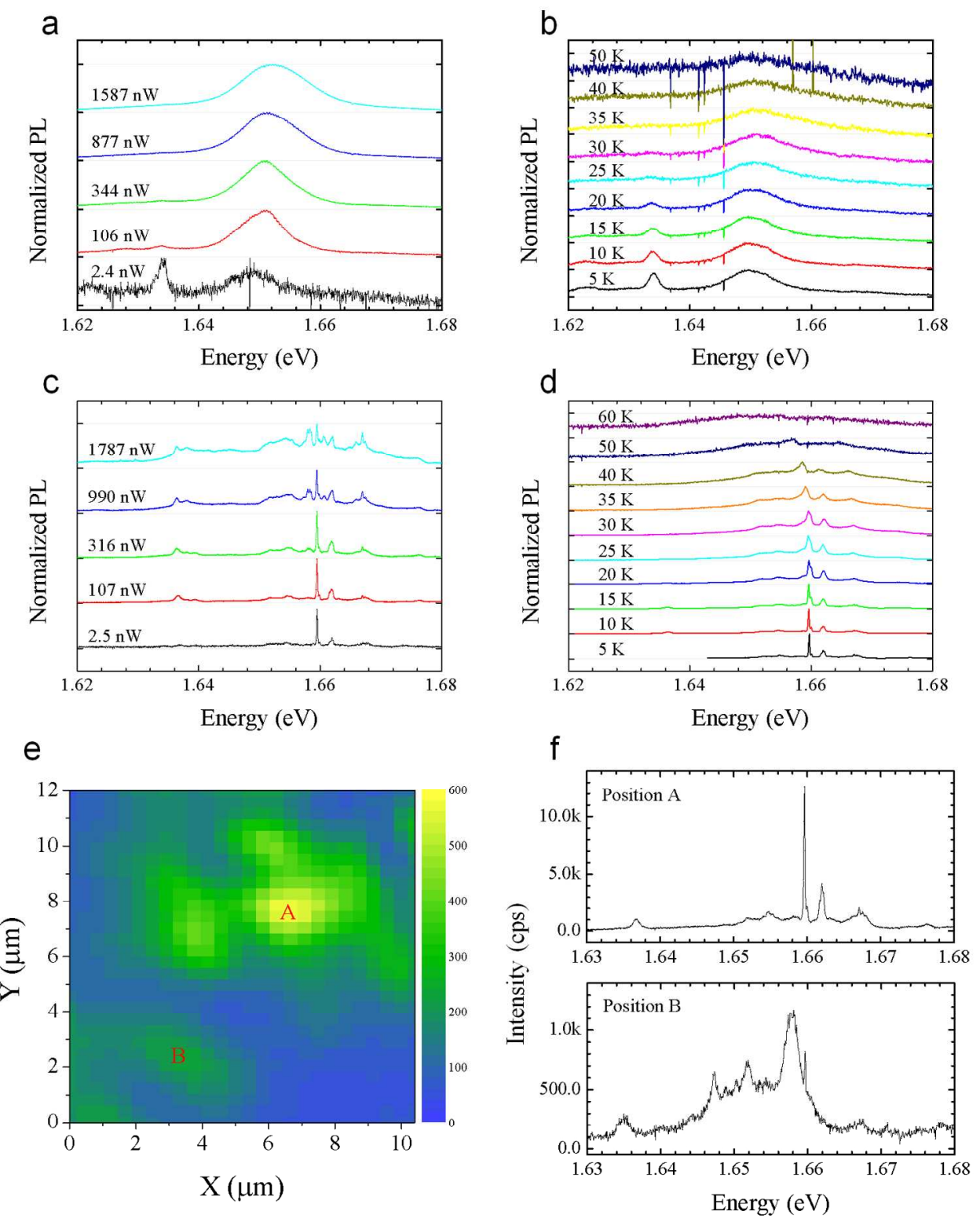

Figure 5

$147 \times 182 \mathrm{~mm}(300 \times 300$ DPI $)$ 